\title{
Determinantes para la elección de los grados de Educación en la Universidad de Murcia
}

María José Orenes Lucas - Universidad de Murcia

0000-0001-8483-6558

Micaela Sánchez Martín - Universidad de Murcia

0000-0002-9107-574X

Recepción: 29.09.2020 | Aceptado: 03.02.2021

Correspondencia a través de ORCID: María José Orenes Lucas

iD 0000-0001-8483-6558

Citar: Orenes Lucas, MJ y Sánchez Martín, M (2021). Determinantes para la elección de los grados de Educación en la Universidad de Murcia. REIDOCREA, 10(9), 1-19.

Resumen: En el presente trabajo se analizan los factores que determinan la elección de los grados de Educación Primaria y Programación Conjunta de Estudios Oficiales de Grado en Educación Infantil y Grado en Educación Primaria (PCEO) en la Facultad de Educación de la Universidad de Murcia. Empleando un enfoque de investigación cuantitativo de diseño descriptivo, los datos han sido recogidos entre 121 estudiantes de primer año de dichas titulaciones, mediante el Cuestionario sobre Determinantes para la Elección del Grado Universitario (CDEGU). Entre los principales resultados y conclusiones de este estudio, cabe destacar que el contexto de los estudiantes de ambas titulaciones es similar, influyendo principalmente el apoyo familiar y social (grupo de pares). En la dimensión determinantes académicos, a pesar de que los estudiantes declaran no haber recibido una orientación suficiente y satisfactoria, obtienen una calificación media de notable en las asignaturas cursadas y muestran una satisfacción muy buena con la elección de los estudios, así como la intención de finalizar la carrera. Respecto a la dimensión determinantes motivacionales, se concluye que los principales motivos que influyen en la elección de la carrera son de carácter intrínseco personal.

Palabra clave: Elección de carrera

Determinants for the choice of the Education degrees at the University of Murcia

Abstract: In this work the factors that determine the choice of Primary Education Degree and Joint Programming of Official Studies of Degree in Infant Education Degree and Primary Education Degree (PCEO) in the Faculty of Education of the University of Murcia are analyzed. Employing a quantitative research approach of descriptive design, the data has been collected among 121 first-year students of these degrees, through the Questionnaire on Determinants for the Choice of the University Degree (CDEGU). Among the main results and conclusions of this study, it should be noted that the context of the students of both degrees is similar, mainly influencing family support and social (peer group). In the academic determinants dimension, despite the fact that the students declare not having received sufficient and satisfactory guidance, obtain an average grade of notable in the subjects taken and show very good satisfaction with the choice of studies, as well as the intention to finish the degree. Regarding the dimension motivational determinants, it is concluded that the main reasons that influence the career choice are intrinsic personal character.

Keyword: Degree choice

\section{Introducción}

La globalización económica de los países europeos ha evolucionado, entre otras causas, por las tecnologías de la información y la comunicación, afectando a todos los aspectos de la vida social. En lo que respecta a la formación, se ha producido un incremento del número de estudiantes que prefieren una formación semipresencial o totalmente virtual que les permita compatibilizar su vida laboral con el aprendizaje (Masjuan, 2005). Además, la actual crisis económica y social y el incremento del paro juvenil ha incrementado la preocupación de este colectivo por su futuro laboral, por lo que continúan formándose en estudios superiores para superar a la competencia existente para la inserción en el mercado laboral (Martínez-Martínez, Castro-Sánchez, Lucena-Zurita, y Zurita-Ortega, 2015). Por consiguiente, se convierte en imprescindible que los modelos de formación, orientación y empleo se adapten al contexto actual 
(Sánchez-Martín, 2020), en el que la orientación debe contribuir a que el alumnado realice una toma de decisiones coherentes con su identidad personal y profesional, conscientes y motivadas (Romero-Rodríguez, 2004).

En este marco, autores como Molina (2004, citado en citado en Martínez Martínez, Castro Sánchez, Zurita Ortega, y Lucena Zurita, 2015) consideran que la orientación laboral es una tarea del sistema educativo. Así pues, identificar los intereses vocacionales de los discentes ayuda en la planificación del proceso de orientación para la elección de la carrera (Sánchez-Martín, Izquierdo, y Contreras, 2017). Aunque este proceso de orientación no permite limitarse a situaciones puntuales. El asesoramiento vocacional va más allá de la elección de estudios o incorporación al mundo laboral, puesto que se trata de un proceso a largo plazo donde intervienen la formación, el autoconocimiento, la representación mental de la realidad sociocultural y la autoconciencia en la toma de decisiones, teniendo en cuenta los componentes sociogénicos y psicogénicos (Rivas, 2003).

Particularmente, la conducta vocacional es definida por Rivas (1988) como "el conjunto de procesos psicológicos que una persona concreta moviliza en relación con el mundo profesional en el que pretende integrarse activamente, o en el que ya está instalado", siendo parte del proceso de socialización (citado en Sánchez-Martín, Izquierdo, y Contreras, 2017, p. 3). Por consiguiente, la conducta vocacional es un trayecto hasta la adultez y atiende a las necesidades sociales y a las de realización personal en el mundo laboral (Rivas, 2003).

Cepero González (2009), por su parte, define las preferencias vocacionales como "respuestas afectivas que da una persona a estímulos ocupacionalmente relevantes", tratándose de la combinación de intereses, aspiraciones, autoevaluaciones y autoconocimiento (p. 13). Mientras que otros autores, como Rocabert (1987) y Rivas (1995), las definen como "el grado de gusto o atracción de la persona por las distintas profesiones" (citado en Cepero González, 2009, p. 16), en la que se combinan aspectos personales, económicos y sociales (Sánchez-Martín, Izquierdo \& Contreras, 2017).

En este proceso de elección, encontramos dos términos vinculados pero distintos: elección y preferencias. Mientras que las preferencias son la variedad de aspectos determinantes por los que la persona tiene atracción, la elección es el resultado final, determinada por el conjunto de preferencias vocacionales y el contexto (Cepero, 2009).

Estudios como los de Echeverría (2010) o Fernández-Tilve y Malvar (2011) muestran que esta decisión ha sido tomada sin indagar en las actitudes, aptitudes y metas personales y profesionales (citado en Martínez-Martínez, et al. 2015), o que la indecisión vocacional puede terminar convirtiéndose en una selección de carreras percibidas como de "menor" esfuerzo y en el aumento de la posibilidad de abandono de esta (GarcíaRipa, Sánchez-García, y Risquez, 2018). Por consiguiente, conocer los determinantes que influyen en la elección sirve para comprenderla mejor y orientar el desarrollo de la carrera e incorporación laboral (Sánchez-Martín, et al., 2017). En este sentido, MartínezMartínez et al. (2015, p. 73) afirman que los resultados sobre los determinantes para la elección del grado proporcionan datos que "pueden ser de utilidad para su uso en el proceso de orientación en esta etapa tan crítica de elección de los adolescentes". Una forma para aplicar los datos recabados sobre los determinantes que influyen en la elección de estudios es la expuesta en la investigación de García-Ripa et, al. (2018), en la que los resultados permitieron elaborar una clasificación de cinco modelos predictivos según las motivaciones y necesidades para diseñar un plan de orientación correspondiente. 
La motivación del alumnado influye en su formación e inserción laboral. Respecto a la dimensión motivacional Masjuan (2005), distingue entre: motivaciones expresivas pertenecientes al sujeto y relacionadas con sus capacidades e intereses; y las motivaciones instrumentales, las cuales hacen referencia a las oportunidades de promocionar en la profesión. Entre los factores que intervienen en la elección de la carrera universitaria Martín-Moreno (1996, citado en Martínez-Martínez et al., 2016, p. 3), diferencia entre intereses intrínsecos: vivencial, vocacional y profesional; y extrínsecos: circunstanciales y fácilmente modificables. Mientras que Castaño (1983, citado en Martínez-Martínez, 2013, p.98) considera que la elección de grado universitario es un proceso que se desarrolla a lo largo de la vida y está compuesta de tres dimensiones:

- Cognitivos: que es el conocimiento de sí mismo con datos objetivos.

- Motivacional: aceptando la sensatez en las aspiraciones.

- Instrumental: en concordancia con los requisitos profesionales y aptitudes personales.

En este sentido y centrándonos en la elección de los estudios de Magisterio GarcíaGarduño (2010, p. 98) considera que "en la elección de la carrera de maestro no sólo intervienen factores ajenos a los propios intereses de los estudiantes, también está basada en la vocación (en la inclinación interna por un oficio o profesión)", además de considerar otros factores como: tener familiares maestros, las expectativas futuras, las expectativas laborales, la capacidad del alumnado, factores económicos, sociales, las salidas profesionales que aporta el grado y la satisfacción del individuo (MartínezMartínez et al., 2015).

En la revisión bibliográfica se han contemplado los principales determinantes que influyen en la elección de estudios en base a la cual nos planteamos la siguiente pregunta de investigación: ¿Cuáles son los factores más relevantes al elegir los grados de Educación Primaria y Programación Conjunta de Estudios Oficiales de Grado en Educación Infantil y Grado en Educación Primaria en la Facultad de Educación de la Universidad de Murcia?

\section{Objetivos}

\section{Objetivo general}

Describir los determinantes para la elección de los grados de Educación Primaria y PCEO en la Facultad de Educación de la Universidad de Murcia.

\section{Objetivos específicos}

1. Identificar qué factores de las características socio-económicas de los estudiantes según la titulación de grado elegida.

2. Conocer los determinantes académicos en la elección de los títulos de grado de Educación Primaria y PCEO.

3. Describir los motivos para la elección de los títulos de grado de Educación Primaria y PCEO.

\section{Métodos}

Este estudio se aborda desde un enfoque cuantitativo, no experimental de corte descriptivo. Este enfoque según Vega-Malagón et al. (2014) pretende responder a las preguntas de investigación mediante el análisis de los datos numéricos recogidos para 
comprobar objetivos e hipótesis previamente establecidos, posee un carácter reduccionista, que intenta generalizar los resultados frente a una población, utilizando muestras representativas y se asocia a instrumentos de recogida de información estandarizados o encuestas de pregunta cerrada. El diseño descriptivo nos permite estudiar un gran número de variables (Abreu, 2012). Por lo que se considera que el método es idóneo debido a la amplia muestra que se pretende recoger, a la gran cantidad de variables que se contemplan y para dar respuesta a los objetivos planteados en este trabajo mediante análisis estadístico descriptivo de datos.

\section{Participantes}

En la planificación inicial de este estudio estaba previsto invitar a todo el alumnado de primer curso de los Grados de Educación Infantil, Educación Primaria y PCEO de la Facultad de Educación de la Universidad de Murcia. Pero este proceso coincidió con la pandemia del Covid-19, la situación de Estado de Alarma y el confinamiento, lo cual obviamente dificultó continuar con dicha planificación. Por lo que finalmente participaron 121 estudiantes de primer curso de las titulaciones de Educación Primaria y PCEO, los cuales fueron seleccionados mediante un muestreo no probabilístico deliberado.

La Tabla 1 muestra las características de los participantes de este estudio, en la que se puede observar que, de los 121 estudiantes, 93 pertenecen al grado de Educación Primaria, con edades compendiadas entre 18 y 26 años $(M=19 ; D T=1.378)$, tratándose de un grupo heterogéneo respecto a la edad. $Y$ no tanto en lo que al sexo se refiere, ya que está ampliamente feminizado con un $74.2 \%$ de mujeres y un $25.8 \%$ hombres. Los 28 estudiantes de PCEO tienen unas edades comprendidas entre los 18 y los 27 años $(M=19 ; D T=1.709)$, tratándose de un grupo más heterogéneo respecto a la edad que el de Educación Primaria y más feminizado con un $89.3 \%$ de mujeres y un $10.7 \%$ hombres.

Respecto al lugar de origen, en ambos grupos la mayoría de los participantes pertenece a distintos municipios de la Región de Murcia y Murcia capital y, en menor medida a provincias vecinas como Alicante, Albacete y Almería.

Tabla 1. Características de la muestra por titulación, sexo, edad y lugar de origen

\begin{tabular}{llll}
\hline Sexo & Primaria, $n=93$ & PCEO, $n=28$ & Total, $n=121$ \\
\hline & $\%$ & $\%$ & $\%$ \\
Hombre & 25.8 & 10.7 & 22.3 \\
Mujer & 74.2 & 89.3 & 77.7 \\
\hline Edad & Primaria, $n=93$ & PCEO, $n=28$ & Total, $n=121$ \\
\hline & $\%$ & $\%$ & $\%$ \\
$17-21$ años & 95.7 & 96.4 & 95.9 \\
$22-26$ años & 4.3 & 0 & 3.3 \\
$27-51$ años & 0 & 3.6 & .8 \\
$M$ & 18.67 & 18.57 & 18.64 \\
$D T$ & 1.378 & 1.709 & 1.454 \\
\hline Lugar de origen & Primaria, $n=93$ & PCEO, $n=28$ & Total, $n=121$ \\
\hline & $\%$ & $\%$ & $\%$ \\
Murcia Municipio & 40.9 & 14.3 & 34.5 \\
Otros municipios de la & 51.1 & 82.1 & 58.6 \\
Región de Murcia & 3.4 & 0 & 2.6 \\
Alicante & 3.4 & 3.6 & 3.4 \\
Albacete & 1.1 & 0 & .9 \\
Almería & & & \\
\hline
\end{tabular}


En relación con el contexto del estudio, los datos fueron recogidos en la Facultad de Educación de la Universidad de Murcia, en la cual se ofrecen los siguientes estudios: cinco grados, nueve masters universitarios, cuatro masters de estudios propios y dos doctorados. Las titulaciones de los grados impartidos son: Educación Infantil, Educación Primaria, Pedagogía, Educación Social y PCEO. El alumnado matriculado en el curso académico 2019-2020 ha sido de 120 estudiantes de Infantil, 363 de Primaria y 62 de PCEO.

\section{Instrumento}

El instrumento de recogida de datos empleado en esta investigación ha sido el Cuestionario sobre Determinantes para la Elección del Grado Universitario (CDEGU), el cual se estructura en tres dimensiones: dimensión socio-económica compuesta por 18 ítems; dimensión determinantes académicos y satisfacción con la elección de la carrera con 11 ítems; y dimensión preferencias vocacionales con 26 ítems de escala tipo Likert; además del apartado inicial sobre datos de identificación.

Respecto a la validez de contenido del instrumento original CDEGU, según SánchezMartín, González, y Martínez, (s.f.), asumiendo un nivel crítico de $p=.05$ en la prueba W de Kendall cinco expertos llegaron a un índice de acuerdo $(p<.05)$ con valores de W $>.5$ en todas las dimensiones que componen el instrumento, presentando una buena validez interna. Por otra parte, la tercera dimensión presenta buenos valores de validez de constructo (con cuatro factores explican el $44.18 \%$ de la varianza total), y de fiabilidad de consistencia interna (con un valor de .741 en el alfa de Cronbach). Concordando este último dato con el cálculo realizado con la muestra de este estudio, con un valor en alfa de Cronbach de .745.

\section{Procedimiento}

El procedimiento para la realización del Trabajo Fin de Máster (TFM) en el que se basa este artículo, se puede estructurar en cuatro fases, las cuales se explican a continuación:

Fase 1. Preliminares. Tras una primera búsqueda bibliográfica se delimitó el tema de investigación, y concretar se planteó el problema y los objetivos de la investigación.

Fase 2. Planificación de la investigación. En la cual se planificó aspectos metodológicos, abordando el problema desde un enfoque cuantitativo. Se decidió, el instrumento de recogida de datos a utilizar y el proceso de análisis de datos. Además, se concretó la técnica de muestreo, solicitando los permisos oportunos de acceso a los participantes para la recogida de información, contactando con los docentes de cada curso de primero de las distintas especialidades de Grados en Maestro de la Facultad de Educación de la Universidad de Murcia.

Fase 3. Trabajo de campo y análisis de datos. En la fase se procedió a la recogida de información, volcado y análisis de los datos. Tras concretar una fecha con los docentes de los cursos de primero que aceptaron la solicitud, empezó el proceso de recogida de información que se efectuó personalmente. A los estudiantes participantes se les informó sobre la naturaleza de la investigación, garantizando la confidencialidad, el anonimato de los datos, la voluntariedad de la participación y resolviendo las dudas que les pudieran surgir al cumplimentar el cuestionario. Los estudiantes dispusieron de 15 minutos para cumplimentar el cuestionario, desarrollándose la recogida de información con normalidad. Al concluir este proceso se agradeció tanto a los participantes como al profesorado su colaboración. 
Fase 4. Redacción del informe. Esta última fase consistió en la redacción final del informe de investigación, siguiendo la normativa del TFM del Máster de Investigación, Evaluación y Calidad en Educación (MIECE).

\section{Análisis de datos}

El análisis de datos se realizó con el paquete estadístico para análisis de datos cuantitativos SPSS versión 24. Para dar respuesta a los objetivos planteados se realizaron análisis estadísticos descriptivos (frecuencia, porcentaje, media y desviación típica).

\section{Resultados}

Para dar respuesta al primer objetivo específico, "Identificar las características socioeconómicas de los estudiantes según la titulación de grado elegida", se realizó un análisis descriptivo de frecuencias de los datos pertenecientes a la dimensión socioeconómica segmentados por titulación.

La Tabla 2 muestra que el $62 \%$ de los participantes tenían cuatro miembros componiendo su unidad familiar, encontrándose principalmente familias con entre tres y seis miembros.

Tabla 2. Componentes de la unidad familiar

\begin{tabular}{llll}
\hline 1) Cuántos miembros componen tu unidad familiar & $\begin{array}{l}\text { Primaria, } \\
n=93\end{array}$ & $\begin{array}{l}\text { PCEO, } \\
n=28\end{array}$ & $\begin{array}{l}\text { Total, } \\
n=121\end{array}$ \\
\hline & $\%$ & $\%$ & $\%$ \\
2 & 2.2 & 0 & 1.7 \\
3 & 20.4 & 10.7 & 18.2 \\
4 & 58.1 & 75.0 & 62.0 \\
5 & 12.9 & 10.7 & 12.4 \\
6 & 3.2 & 3.6 & 3.3 \\
7 & 1.1 & 0 & .8 \\
9 & 1.1 & 0 & .8 \\
10 & 1.1 & 0 & .8 \\
\hline
\end{tabular}

Como se puede observar en la Tabla 3, el $62.8 \%$ de esas familias contaban con dos miembros trabajando, habiendo casos más extremos de algunas familias sin ningún miembro trabajando y otras con hasta seis miembros.

Tabla 3. Miembros de la unidad familiar empleados

\begin{tabular}{llll}
\hline 2) Cuantos miembros de tu unidad familiar trabajan & $\begin{array}{l}\text { Primaria, } \\
n=93\end{array}$ & $\begin{array}{l}\text { PCEO, } \\
n=28\end{array}$ & $\begin{array}{l}\text { Total, } \\
n=121\end{array}$ \\
\hline & $\%$ & $\%$ & $\%$ \\
0 & 2.2 & 3.6 & 2.5 \\
1 & 23.7 & 17.9 & 22.3 \\
2 & 59.1 & 75.0 & 62.8 \\
3 & 11.8 & 3.6 & 9.9 \\
4 & 1.1 & 0 & .8 \\
5 & 1.1 & 0 & .8 \\
6 & 1.1 & 0 & .8 \\
\hline
\end{tabular}


Respecto a la vivencia actual, en la Tabla 4, se muestra que, en Primaria, el $79.3 \%$ de la muestra vivía con la familia y que, el resto de este grupo, estaba distribuido entre amigos/as, pareja, solo/a o con la familia y amigos/as. Mientras que en PCEO, el 51.9 $\%$ de la muestra vivía con amigos/as y el resto con la familia.

Tabla 4. Vivencia actual

\begin{tabular}{llll}
\hline 3) Con quién vives actualmente & Primaria, $n=93$ & PCEO, $n=28$ & Total, $n=121$ \\
\hline & $\%$ & $\%$ & $\%$ \\
Familia & 79.3 & 48.1 & 72.3 \\
Amigos/as & 14.1 & 51.9 & 22.7 \\
Pareja & 3.3 & 0 & 2.5 \\
Solo/a & 1.1 & 0 & .8 \\
Familia y amigos/as & 2.2 & 0 & 1.7 \\
\hline
\end{tabular}

La Tabla 5 recoge el nivel de estudios de los progenitores, en Primaria el $30.8 \%$ de los padres poseía un nivel de estudios de grado y el resto F.P. de grado superior, estudios secundarios, estudios primarios y máster o doctorado, existiendo un $2.2 \%$ que no poseían estudios. En PCEO, el 33.3 \% poseía un nivel de estudios secundarios y el resto F.P de grado superior, grado, estudios primarios y máster o doctorado. El $43.7 \%$ de las madres de los participantes poseían un nivel de estudios de grado, existiendo porcentajes similares en los estudios secundarios y de F.P. de grado superior, encontrando el doble de madres de los estudiantes de PCEO que en Primaria con estudios primarios y un $5.4 \%$ de las de Primaria con estudios de máster o doctorado, cuando en PCEO ninguna madre posee este nivel de estudios.

Tabla 5. Nivel de estudios de los progenitores

\begin{tabular}{llll}
\hline 4) Nivel de estudios del padre & Primaria, $n=93$ & PCEO, $n=28$ & Total, $n=121$ \\
\hline Sin estudios & $\%$ & $\%$ & $\%$ \\
Estudios primarios & 2.2 & 0 & 1.7 \\
Estudios Secundarios & 11.0 & 18.5 & 12.7 \\
F.P. Grado Superior & 20.9 & 33.3 & 23.7 \\
Grado & 26.4 & 22.2 & 25.4 \\
Máster/Doctorado & 30.8 & 22.2 & 28.8 \\
\hline 5) Nivel de estudios de la madre & 8.8 & 3.7 & 7.6 \\
\hline & Primaria, $n=93$ & PCEO, $n=28$ & Total, $n=121$ \\
Estudios primarios & $\%$ & $\%$ & $\%$ \\
Estudios Secundarios & 9.8 & 18.5 & 11.8 \\
F.P. Grado Superior & 23.9 & 22.2 & 23.5 \\
Grado & 16.3 & 18.5 & 16.8 \\
Máster/Doctorado & 44.6 & 40.7 & 43.7 \\
\hline
\end{tabular}

En cuanto a la situación laboral de los progenitores, como muestra la Tabla 6, la mayoría de los padres trabajaban (88 \%), encontrándose casos de padres jubilados (5.1\%) y parados $(6.8 \%)$, respecto a las madres trabajaban un $69.2 \%$, difiriendo los porcentajes entre las titulaciones respecto a madres paradas y con trabajo doméstico, siendo más altos en Educación Primaria, y porcentajes similares entre los grupos de las madres de los estudiantes que estaban jubiladas. 
Tabla 6. Situación laboral de los progenitores

\begin{tabular}{llll}
\hline 6) Trabajo del padre & Primaria, $n=93$ & PCEO, $n=28$ & Total, $n=121$ \\
\hline & $\%$ & $\%$ & $\%$ \\
Trabaja & 87.8 & 88.9 & 88.0 \\
Jubilado & 5.6 & 3.7 & 5.1 \\
Parado & 6.7 & 7.4 & 6.8 \\
\hline 7) Trabajo de la madre & Primaria, $n=93$ & PCEO, $n=28$ & Total, $n=121$ \\
\hline & $\%$ & $\%$ & $\%$ \\
Trabaja & 66.7 & 77.8 & 69.2 \\
Jubilada & 2.2 & 3.7 & 2.5 \\
Parada & 7.5 & 3.7 & 6.7 \\
Trabajo doméstico & 23.7 & 14.8 & 21.7 \\
\hline
\end{tabular}

En lo referente a datos económicos, los ingresos mensuales de la unidad familiar (Tabla 7), el $52.1 \%$ de los participantes tenían unos ingresos de menos de $1000 €$ y el $20.7 \%$ de más de $3000 €$.

Tabla 7. Ingresos familiares

\begin{tabular}{llll}
\hline 8) Ingresos mensuales de la unidad familiar & $\begin{array}{l}\text { Primaria, } \\
n=93\end{array}$ & $\begin{array}{l}\text { PCEO, } n= \\
28\end{array}$ & Total, $n=121$ \\
\hline & $\%$ & $\%$ & $\%$ \\
Menos de $1000 €$ & 53.8 & 46.4 & 52.1 \\
De 1001 a $1500 €$ & 5.4 & 14.3 & 7.4 \\
De 1501 a $2000 €$ & 12.9 & 7.1 & 11.6 \\
De 2001 a 2500€ & 6.5 & 7.1 & 6.6 \\
De 2501 a 2999€ & 2.2 & 0 & 1.7 \\
Más de $3000 €$ & 19.4 & 25.0 & 20.7 \\
\hline
\end{tabular}

Como se observa en la Tabla 8 , más de la mitad de los participantes del grupo de Primaria continúan viviendo en el mismo lugar $(68.5 \%)$ y la mitad de PCEO (50\%) tuvieron que cambiar de ciudad para cursar la titulación elegida.

Tabla 8. Cambio de ciudad

\begin{tabular}{llll}
\hline 9) ¿Cambiaste de ciudad para cursar esta titulación? & $\begin{array}{l}\text { Primaria, } \\
n=93\end{array}$ & PCEO, $n=28$ & Total, $n=121$ \\
\hline & $\%$ & $\%$ & $\%$ \\
Si & 31.5 & 50.0 & 35.7 \\
No & 68.5 & 50.0 & 64.3 \\
\hline
\end{tabular}

Los datos sobre la financiación de estudios de los estudiantes se recogen en la Tabla 9. En cuanto a la situación laboral, en ambos grupos la mayoría de los participantes (86.8 $\%)$ no trabajaban en ese momento. Asimismo, la mayoría de los estudiantes (90.8\%) recibe apoyo económico de la familia. Respecto a las becas, recibían más becas los estudiantes de PCEO (71.4\%) que los de Primaria, con un $51.6 \%$ de participantes que no recibían beca. 
Tabla 9. Financiación de los estudios

\begin{tabular}{llll}
\hline 10) ¿Trabajas actualmente? & Primaria, $n=93$ & PCEO, $n=28$ & Total, $n=121$ \\
\hline \multirow{2}{*}{$\mathrm{Si}$} & $\%$ & $\%$ & $\%$ \\
$\mathrm{No}$ & 15.1 & 7.1 & 13.2 \\
\hline 11) Recibe apoyo económico de la familia & Primaria, $n=93$ & PCEO, $n=28$ & Total, $n=121$ \\
\hline & $\%$ & $\%$ & $\%$ \\
$\mathrm{Si}$ & 89.1 & 96.4 & 90.8 \\
$\mathrm{No}$ & 10.9 & 3.6 & 9.2 \\
\hline 12) Recibe beca & Primaria, $n=93$ & PCEO, $n=28$ & Total, $n=121$ \\
\hline & $\%$ & $\%$ & $\%$ \\
$\mathrm{Si}$ & 48.4 & 71.4 & 53.7 \\
No & 51.6 & 28.6 & 46.3 \\
\hline
\end{tabular}

En cuanto a la influencia sociofamiliar, como se observa en la Tabla 10, la mayoría de la muestra no estudia la titulación por tradición familiar $(66.9 \%)$. El $97.5 \%$ de los participantes contaban con el apoyo de sus padres para estudiar la carrera, encontrando algunos casos puntuales en Primaria donde el $3.3 \%$ de los participantes no recibían dicho apoyo. Asimismo, hubo más casos de estudiantes con amigos que habían estudiado o tenían la intención de estudiar esa carrera en PCEO (85.7\%) que en Primaria $(64.5 \%)$. La pareja no parece un determinante que influenció esta elección, ya que el $92.3 \%$ de los estudiantes que tenían pareja en el momento de elegir la carrera, no habían estudiado esta carrera ni tenían intención de hacerlo.

Tabla 10. Influencia socio familiar

\begin{tabular}{llll}
\hline 13) Algún miembro de su familia cursó esta carrera & Primaria, & PCEO, & Total, \\
$n=93$ & $n=28$ & $n=121$ \\
\hline & $\%$ & $\%$ & $\%$ \\
$\mathrm{Si}$ & 35.5 & 25.0 & 33.1 \\
$\mathrm{No}$ & 64.5 & 75.0 & 66.9 \\
\hline 14) Tus padres piensan que esta carrera es adecuada para ti & Primaria, & PCEO, & Total, \\
& $n=93$ & $n=28$ & $n=121$ \\
\hline & $\%$ & $\%$ & $\%$ \\
$\mathrm{Si}$ & 96.7 & 100.0 & 97.5 \\
$\mathrm{No}$ & 3.3 & 0 & 2.5 \\
\hline 15) ¿Algunos de tus amigos estudiaban o tenían intención de & Primaria, & PCEO, & Total, \\
estudiar esta titulación? & $n=93$ & $n=28$ & $n=121$ \\
\hline & $\%$ & $\%$ & $\%$ \\
Si & 64.5 & 85.7 & 69.4 \\
No & 35.5 & 14.3 & 30.6 \\
\hline 16) ¿Tenías pareja en el momento de la elección de la & Primaria, & PCEO, & Total, \\
carrera? & $n=93$ & $n=28$ & $n=121$ \\
\hline & $\%$ & $\%$ & $\%$ \\
Si & 41.9 & 46.4 & 43.0 \\
No & 58.1 & 53.6 & 57.0 \\
\hline 17) ¿Tu pareja estudiaba o tenía intención de estudiar lo & Primaria, & PCEO, & Total, \\
mismo que tú? & $n=93$ & $n=28$ & $n=121$ \\
\hline & $\%$ & $\%$ & $\%$ \\
Si & 6.5 & 12.5 & 7.7 \\
No & 93.5 & 87.5 & 92.3 \\
\hline
\end{tabular}


En la Tabla 11, se observa que el $95 \%$ de los estudiantes de estos grados consideraban que no existe existen carreras más adecuadas para mujeres y para hombres, lo que sugiere que la adecuación al género no fue un determinante para elegir estas titulaciones.

Tabla 11. Adecuación de la carrera al género

\begin{tabular}{llll}
\hline 18$)$ ¿Crees que existen carreras más adecuadas para hombre & Primaria, & PCEO, & Total, \\
y carreras más adecuadas para mujeres? & $n=93$ & $n=28$ & $n=121$ \\
\hline & $\%$ & $\%$ & $\%$ \\
$\mathrm{Si}$ & 6.5 & 0 & 5.0 \\
$\mathrm{No}$ & 93.5 & 100.0 & 95.0 \\
\hline
\end{tabular}

Para despejar el segundo objetivo, "Conocer los determinantes académicos en la elección de los títulos de grado de Educación Primaria y PCEO", se realizó un análisis descriptivo de frecuencias de los datos pertenecientes a la dimensión determinantes académicos y satisfacción con la elección de la carrera.

Así, la Tabla 12 muestra, que la mayoría del alumnado (95 \%) accedió al grado por Bachillerato, encontrándose casos puntuales de acceso por Grado Superior y mayores de 25 en el grupo de Primaria y por otra titulación en PCEO.

Tabla 12. Acceso al grado

\begin{tabular}{llll}
\hline 1) Como accediste a la carrera universitaria & Primaria, $n=93$ & PCEO, $n=28$ & Total, $n=121$ \\
\hline & $\%$ & $\%$ & $\%$ \\
Bachillerato & 94.6 & 96.4 & 95.0 \\
Grado Superior & 4.3 & 0 & 3.3 \\
Mayores 25 & 1.1 & 0 & .8 \\
Otra titulación & 0 & 3.6 & .8 \\
\hline
\end{tabular}

Respecto al rendimiento académico, en la Tabla 13 se observan diferencias de porcentaje en las notas medias entre las titulaciones. En Primaria, el $47.8 \%$ tenía una nota de entre 9 y 10.99 , el $44.4 \%$ entre 11 y 12.99 y el resto entre 7 y 8.99 o entre 13 y 14. En PCEO, el $82.1 \%$ tenía una nota de entre 11 y 12.99 , el $14.3 \%$ de entre 13 y 14 y el resto de entre 7 y 8.99 . Estos resultados vienen a sugerir que los participantes de PCEO poseen una media superior a los de Primaria. También, concuerdan con el rendimiento académico en la nota media de la titulación. Los estudiantes de PCEO poseían notas más altas, teniendo un notable el $72 \%$ de los participantes de este grupo, el $16 \%$ un sobresaliente y el resto una calificación de bien. Mientras que en Primaria el $62.2 \%$ tenían una calificación de notable, el $25.6 \%$ de bien y el resto (12.2 \%) engloban calificaciones de sobresaliente, aprobado o insuficiente. 
Tabla 13. Rendimiento académico

\begin{tabular}{llll}
\hline 2) Nota media de acceso al Grado & Primaria, $n=$ & PCEO, $n=28$ & Total, $n=121$ \\
\hline & 93 & $\%$ & $\%$ \\
$7-8.999$ & $\%$ & 3.6 & 3.4 \\
$9-10.999$ & 3.3 & 0 & 36.4 \\
$11-12.999$ & 47.8 & 82.1 & 53.4 \\
$13-14$ & 44.4 & 14.3 & 6.8 \\
\hline 3) Nota media de las asignaturas cursadas & Primaria, $n=93$ & PCEO, $n=28$ & Total, $n=121$ \\
\hline & $\% .4$ & $\%$ & $\%$ \\
Insuficiente & 1.2 & 0 & .9 \\
Aprobado & 6.1 & 0 & 4.7 \\
Bien & 25.6 & 12.0 & 22.4 \\
Notable & 62.2 & 72.0 & 64.5 \\
Sobresaliente & 4.9 & 16.0 & 7.5 \\
\hline
\end{tabular}

En preferencia al número de opción de elección de la carrera (Tabla 14), la mayoría de los participantes $(82.6 \%)$ eligieron estas titulaciones como primera opción, pudiendo encontrar casos en el grupo de Primaria que la eligieron como segunda o tercera opción y en PCEO casos en los que seleccionaron esta titulación como segunda opción. En cuanto a los motivos para no elegirla como primera opción en Primaria fue por: no haber plazas, pocas salidas profesionales, no hallarse en su ciudad, dudas o por cursar un año y decidir cambiar de grado al año siguiente. Mientras que en PCEO los motivos de los estudiantes para no elegir la titulación como primera opción fueron por no tener plaza en la carrera deseada y por dudas o indecisión.

Tabla 14. Preferencia en la elección

\begin{tabular}{llll}
\hline 4) Número de opción en el que elegiste esta carrera & $\begin{array}{l}\text { Primaria, } \\
n=93\end{array}$ & $\begin{array}{l}\text { PCEO, } \\
n=28\end{array}$ & $\begin{array}{l}\text { Total, } \\
n=121\end{array}$ \\
\hline & $\%$ & $\%$ & $\%$ \\
1 & 79.6 & 92.9 & 82.6 \\
2 & 18.3 & 7.1 & 15.7 \\
3 & 2.2 & 0 & 1.7 \\
\hline 5) Motivos por los que no cursaste la carrera elegida en primera & Primaria, & PCEO, & Total, \\
opción & $n=93$ & $n=28$ & $n=121$ \\
\hline & $\%$ & $\%$ & $\%$ \\
Es la que quería & 79.6 & 92.9 & 82.6 \\
Pocas salidas profesionales & 2.2 & 0 & 1.7 \\
No había en esta ciudad & 2.2 & 0 & 1.7 \\
No había plaza & 14.0 & 3.6 & 11.6 \\
Dudaba & 1.1 & 3.6 & 1.7 \\
La cursó un año, pero decidió cambiar de grado al año siguiente & 1.1 & 0 & .8
\end{tabular}

En la Tabla 15 se recogen los datos sobre la orientación previa recibida para la elección de los estudios. En la titulación de Primaria, la mayoría de los estudiantes (54.4 \%) dicen que se les proporcionó información sobre los grados únicamente en su Centro, el 33.3 $\%$ la obtuvieron de forma autónoma por internet y en menor medida a través de: Centro donde estudiaron e internet $(4.4 \%)$, Centro de Orientación (3.3\%), familia (1.1\%) o Centro donde estudiaron internet y familia (1.1\%); existiendo un $2.2 \%$ de estudiantes 
que declaran no haber recibido ningún tipo de orientación previa para realizar la elección de la carrera.

Los principales medios por donde obtuvieron la información sobre las titulaciones los estudiantes de PCEO fueron el Centro donde estudiaron $(39.3 \%)$ exclusivamente e internet (39.3\%). El resto de los participantes de PCEO consiguió dicha información por: el Centro donde estudiaron e internet (10.7\%), la Universidad de Murcia (3.6\%), la familia y universidad (3.6\%) o el Centro donde estudiaron y universidad (3.6 \%). Aunque la mayoría de los discentes recibieron información sobre los grados en sus Centros, el $61.2 \%$ percibían no haber recibido suficiente información sobre los planes de estudios. Asimismo, el $65.8 \%$ de los participantes no realizaron una prueba para conocer qué carrera se adaptaba más a sus habilidades y capacidades.

Tabla 15. Orientación para la elección de estudios

\begin{tabular}{llll}
\hline 6) ¿Dónde te proporcionaron información sobre los planes de & Primaria, & PCEO, & Total, \\
Grado & $n=93$ & $n=28$ & $n=121$ \\
\hline & $\%$ & $\%$ & $\%$ \\
Centro donde estudie & 54.4 & 39.3 & 50.8 \\
Internet & 33.3 & 39.3 & 34.7 \\
Centro de Orientación & 3.3 & 0 & 2.5 \\
Centro donde estudie e internet & 4.4 & 10.7 & 5.9 \\
Universidad de Murcia & 0 & 3.6 & .8 \\
Familia y universidad & 0 & 3.6 & .8 \\
Centro donde estudie y universidad & 0 & 3.6 & .8 \\
No se la proporcionaron & 2.2 & 0 & 1.7 \\
Centro donde estudie, internet y familia & 1.1 & 0 & .8 \\
Familia & 1.1 & 0 & .8 \\
\hline 7) Información suficiente sobre planes de estudios & Primaria, & PCEO, & Total, \\
\hline & $n=93$ & $n=28$ & $n=121$ \\
\hline Si & $\%$ & $\%$ & $\%$ \\
No & 40.9 & 32.1 & 38.8 \\
\hline 8) Has realizado alguna prueba para conocer qué carrera se & Primaria, & PCEO, \\
adapta más a tus habilidades y capacidades & $n=93$ & $n=28$ & $n=121$ \\
\hline & $\%$ & $\%$ & $\%$ \\
Si & 34.8 & 32.1 & 34.2 \\
No & 65.2 & 67.9 & 65.8 \\
\hline
\end{tabular}

La satisfacción con la orientación recibida para la elección de los grados (Tabla 16) fue escasa para el $42.5 \%$ de la muestra, suficiente para el $30.8 \%$, buena para el $20.8 \%$, encontrando una minoría de participantes que la considero nula o muy buena.

Tabla 16. Satisfacción con la orientación para la elección

\begin{tabular}{llll}
\hline 9) Satisfacción con la información previa para la elección del & $\begin{array}{l}\text { Primaria, } \\
n=93\end{array}$ & $\begin{array}{l}\text { PCEO, } \\
n=28\end{array}$ & $\begin{array}{l}\text { Total, } \\
\text { Grado }\end{array}$ \\
\hline & $\%$ & $\%$ & $\%$ \\
Nula & 2.2 & 3.6 & 2.5 \\
Escasa & 41.3 & 46.4 & 42.5 \\
Suficiente & 30.4 & 32.1 & 30.8 \\
Buena & 22.8 & 14.3 & 20.8 \\
Muy buena & 3.3 & 3.6 & 3.3 \\
\hline
\end{tabular}


Como se observa en la Tabla 17, la satisfacción con el Grado fue muy buena para el $50.4 \%$ de la muestra, buena para el $35.5 \%$ y suficiente para el $12.4 \%$, siendo escasa para una minoría de participantes de la titulación de Primaria.

Tabla 17. Satisfacción con la elección del grado

\begin{tabular}{llll}
\hline $\begin{array}{l}\text { 10) Grado de satisfacción con la elección del } \\
\text { grado }\end{array}$ & $\begin{array}{l}\text { Primaria, } \\
n=93\end{array}$ & PCEO, $n=28$ & Total, $n=121$ \\
\hline & $\%$ & $\%$ & $\%$ \\
Escasa & 2.2 & 0 & 1.7 \\
Suficiente & 14.0 & 7.1 & 12.4 \\
Buena & 35.5 & 35.7 & 35.5 \\
Muy buena & 48.4 & 57.1 & 50.4 \\
\hline
\end{tabular}

En cuanto a los planes de futuro de los estudiantes (Tabla 18), el $83.5 \%$ de los participantes tenían planeado terminar la carrera. En la titulación de Primaria, las otras perspectivas para los próximos años eran: terminar la carrera alternándola con el trabajo, ningún plan, trabajar o cambiar de carrera, terminar superior de piano y la carrera o dejar la carrera y hacer un Grado Superior. Mientras en la titulación de PCEO el otro plan de futuro fue terminar la carrera alternándola con el trabajo.

Tabla 18. Planes de futuro

\begin{tabular}{llll}
\hline 11) Perspectivas para los próximos años & Primaria, $n=93$ & PCEO, $n=28$ & Total, $n=121$ \\
\hline & $\%$ & $\%$ & $\%$ \\
Terminar la carrera & 81.7 & 89.3 & 83.5 \\
Trabajar & 1.1 & 0 & .8 \\
Terminar la carrera y trabajar & 10.8 & 10.7 & 10.7 \\
No tiene & 3.2 & 0 & 2.5 \\
Terminar superior de piano y la carrera & 1.1 & 0 & .8 \\
Cambiar la carrera o terminarla & 1.1 & 0 & .8 \\
Dejar la carrera y hacer un grado superior & 1.1 & 0 & .8 \\
\hline
\end{tabular}

Para responder al tercer objetivo "Describir los motivos para la elección de los títulos de grado de Educación Primaria y PCEO", se realizó un análisis descriptivo de los datos pertenecientes a la dimensión preferencias vocacionales. Los motivos para la elección de los grados pueden ser de cuatro tipos: personales de carácter intrínseco, instrumentales de carácter extrínseco, profesionales de carácter extrínseco y profesionales de carácter intrínseco.

Los motivos personales de carácter intrínseco se recogen en la Tabla 19. Los participantes respondieron con una media entre 4.31 y 4.96 a cada uno de los ítems correspondientes con este tipo de motivos. Asimismo, en cada ítem las respuestas de los participantes de cada titulación fueron similares. La desviación típica inferior a 1, señala la homogeneidad de los datos. Lo cual muestra la relevancia de este tipo de motivos de los participantes para la elección de las titulaciones de Educación Primaria y PCEO. 
Tabla 19. Motivos personales de carácter intrínseco

\begin{tabular}{lllll}
\hline $\begin{array}{l}\text { Motivos para la elección de los estudios. Personales de carácter } \\
\text { intrínseco }\end{array}$ & $\begin{array}{l}\text { Primaria, } \\
n=93\end{array}$ & \multicolumn{2}{l}{$\begin{array}{l}\text { PCEO, } \\
n=28\end{array}$} \\
\cline { 2 - 5 } & $M$ & $D T$ & $M$ & $D T$ \\
\hline 1) Importante desarrollar capacidades personales en la profesión & 4.70 & .586 & 4.89 & .315 \\
2) Importante estar en constante crecimiento personal & 4.66 & .634 & 4.82 & .390 \\
3) Importante superarme constantemente como persona en el trabajo & 4.57 & .682 & 4.82 & .476 \\
4) Importante que mi carrera me permita estar a gusto conmigo mismo & 4.81 & .495 & 4.96 & .189 \\
5) Importante que las personas de mi alrededor estén contentas con & 4.46 & .834 & 4.36 & .911 \\
ellas mismas & & & & \\
6) Importante ayudar a los demás a desarrollarse personal y & 4.31 & .780 & 4.68 & .548 \\
profesionalmente & & & & \\
7) Es importante conseguir metas & 4.37 & .734 & 4.54 & .637 \\
8) Importante buscar alternativas cuando no se resuelve un problema & 4.51 & .689 & 4.68 & .548 \\
9) Para mi es importante explorar distintas actividades & 4.18 & .769 & 4.25 & .844 \\
\hline
\end{tabular}

En cuanto a los motivos instrumentales de carácter extrínseco (Tabla 20), en el grupo de Primaria la media de respuesta al ítem 10 "con esta carrera voy a conseguir ingresos seguros" fue de 3.29, pero la desviación típica muestra la heterogeneidad de los datos, al ser superior al valor 1 . Aunque la media de respuestas en este ítem es la misma en PCEO, en este último grupo los datos son homogéneos. En el ítem 11 "carrera con buenas salidas profesionales", puntuaciones medias de respuestas en ambos grupos fueron de 3.66 (Primaria) y 3.82 (PCEO), sin embargo los datos de Primaria fueron heterogéneos y los de PCEO homogéneos. Respecto al ítem 12 "me aportará prestigio profesional", la media de respuesta fue 3.33 (Primaria) y 3.18 (PCEO), siendo los datos homogéneos en el grupo de Primaria y heterogéneos en el de PCEO. La puntuación media para el ítem 13 "es importante tener personal bajo mi cargo" fue de 2.39 (Primaria) y 2.54 (PCEO), siendo heterogéneas en los participantes de Primaria y heterogéneas en los de PCEO. Para los ítems 14 "el prestigio y un puesto directivo es importante", 15 "considero importante ser un líder" y 16 "es importante conseguir ascensos a largo plazo" las medias de las respuestas fueron similares entre titulaciones y en ambos grupos heterogéneas. En cuanto al ítem 17 "para mi es importante ganar mucho dinero", las medias de las puntuaciones fueron de 3.04 (Primaria) y 2.89 (PCEO), siendo los datos de Primaria heterogéneos y los de PCEO homogéneos. Teniendo en cuenta estos resultados, se puede suponer que los motivos intrínsecos instrumentales poseen menor peso en la elección de estas carreras.

\section{Tabla 20. Motivos instrumentales de carácter extrínseco}

\begin{tabular}{lllll}
\hline Motivos para la elección de los estudios. Instrumentales de & \multicolumn{3}{l}{ Primaria, $n=93$} & \multicolumn{2}{l}{ PCEO, $n=28$} \\
\cline { 2 - 5 } carácter extrínseco & $M$ & $D T$ & $M$ & $D T$ \\
\hline 10) Con esta carrera voy a conseguir ingresos seguros & 3.29 & 1.125 & 3.29 & .937 \\
11) Carrera con buenas salidas profesionales & 3.66 & 1.128 & 3.82 & .863 \\
12) Me aportará prestigio profesional & 3.33 & .978 & 3.18 & 1.090 \\
13) Es importante tener personal bajo mi cargo & 2.39 & 1.138 & 2.54 & .999 \\
14) El prestigio y un puesto directivo es importante & 2.88 & 1.098 & 3.04 & 1.170 \\
15) Considero importante ser un líder & 2.82 & 1.109 & 2.71 & 1.150 \\
16) Es importante conseguir ascensos a largo plazo & 3.16 & 1.035 & 3.37 & 1.043 \\
17) Para mi es importante ganar mucho dinero & 3.04 & 1.058 & 2.89 & .916 \\
\hline
\end{tabular}

Respecto a los motivos profesionales de carácter extrínseco, en la Tabla 21 se observa que en el ítem 18 "es importante influir en la opinión de los demás" la media de las puntuaciones de las titulaciones fue $\operatorname{similar}(M=2.50$ y $D T=1)$, siendo respuestas heterogéneas en ambas titulaciones. Las puntuaciones para el ítem 19 "me parece importante convencer a la gente con mis ideas" fueron similares en los dos grupos de participantes, sin embargo, en el grupo de Primaria los datos eran homogéneos y en el 
de PCEO heterogéneos. Para los ítems 20 "es importante recibir reconocimiento por los conocimientos en los que me he especializado", 21 "especializarse en área específica es importante", 22 "para mi es importante trabajar en temas especializados" y 23 "seguir procedimientos seguros y oportunos es importante" la media de respuestas en cada ítem fue similar entre las titulaciones y las puntuaciones homogéneas dentro del mismo grupo de grado. De esta manera, se puede suponer que estos motivos tuvieron menor peso en la elección de estos grados.

Tabla 21. Motivos profesionales de carácter extrínseco

\begin{tabular}{lllll}
\hline $\begin{array}{l}\text { Motivos para la elección de los estudios. Profesionales de carácter } \\
\text { extrínseco }\end{array}$ & $\begin{array}{l}\text { Primaria, } \\
n=93\end{array}$ & \multicolumn{2}{l}{ PCEO, $n=28$} \\
\cline { 2 - 5 } & $M$ & $D T$ & $M$ & $D T$ \\
\hline 18) Es importante influir en la opinión de los demás & 2.91 & 1.090 & 2.50 & 1.000 \\
19) Me parece importante convencer a la gente con mis ideas & 3.34 & .991 & 3.07 & 1.152 \\
20) Es importante recibir reconocimiento por los conocimientos en los & 3.92 & .912 & 4.00 & .770 \\
que me he especializado & 3.79 & .955 & 3.64 & .826 \\
21) Especializarse en área específica es importante & 3.69 & .897 & 3.21 & .568 \\
22) Para mi es importante trabajar en temas especializados & 3.82 & .765 & 4.07 & .604 \\
23) Seguir procedimientos seguros y oportunos es importante & & & & \\
\hline
\end{tabular}

Respecto a los datos sobre los motivos profesionales de carácter intrínseco (Tabla 22), la media de las puntuaciones del ítem 24 "trabajar sin preocuparme de las restricciones institucionales o empresariales" fueron 3.70 (Primaria) y 3.39 (PCEO), siendo las respuestas de Primaria homogéneas y las de PCEO heterogéneas. En cuanto al ítem 25 "trabajar sin que me supervisen estrictamente", la media de las puntuaciones en Primaria fue de 3.95 siendo un grupo por sus datos heterogéneo, mientras que en PCEO fue de 3.43 , siendo un grupo por sus respuestas homogéneo. Respecto al ítem 26 "que no haya normas ni regulaciones estrictas en mi trabajo", la media de las puntuaciones fue 3.08 (Primaria) y 2.36 (PCEO), y aunque ambos grupos son heterogéneos por sus datos, el grupo de Primaria es más heterogéneo que el de PCEO.

Tabla 22. Motivos profesionales de carácter intrínseco

\begin{tabular}{|c|c|c|c|c|}
\hline \multirow[t]{2}{*}{$\begin{array}{l}\text { Motivos para la elección de los estudios. Profesionales de carácter } \\
\text { intrínseco }\end{array}$} & \multicolumn{2}{|c|}{$\begin{array}{l}\text { Primaria, } \\
n=93\end{array}$} & \multicolumn{2}{|c|}{ PCEO, $n=28$} \\
\hline & M & $D T$ & M & $D T$ \\
\hline $\begin{array}{l}\text { 24) Trabajar sin preocuparme de las restricciones institucionales o } \\
\text { empresariales }\end{array}$ & 3.70 & .998 & 3.39 & 1.166 \\
\hline 25) Trabajar sin que me supervisen estrictamente & 3.95 & 1.097 & 3.43 & .742 \\
\hline 26) Que no haya normas ni regulaciones estrictas en mi trabajo & 3.08 & 1.227 & 2.36 & 1.162 \\
\hline
\end{tabular}

\section{Discusión}

Con los resultados del primer objetivo "Identificar qué factores de las características socioeconómicas de los estudiantes según la titulación de grado elegida" podemos concluir que:

Las familias de los participantes poseen unas características socioeconómicas similares. En general, la mayoría de los estudiantes continúan viviendo en la unidad familiar, compuesta principalmente de cuatro miembros, en la que dos de ellos trabajan. El nivel de estudios de los padres se agrupa principalmente en los rangos de Estudios de Secundaria y Formación Profesional de Grado Superior, mientras que las madres han estudiado, en su mayor parte, Formación Profesional de Grado Superior y Grados Universitarios. Respecto a la financiación de los estudios el alumnado de ambos grupos no trabaja, proviniendo su principal fuente de ingresos de la familia, seguido de la ayuda 
institucional. En este punto cabe destacar que los estudiantes de PCEO reciben mayor número de becas que los estudiantes de Educación Primaria.

Respecto a la influencia sociofamiliar, en la elección de estas titulaciones, la familia, la pareja y los estereotipos de género, no son factores que determinen la elección de los estudios de estos grados de educación. Datos que no se corresponden con los obtenidos por García Garduño (2010), quien afirma que uno de los factores determinantes en la elección de los estudios de educación es tener familiares maestros. Sin embargo, tanto la aprobación familiar como la influencia del grupo de iguales sí son determinantes relevantes dicha elección. Siendo el grupo de amigos el determinante con más peso en la elección, dato que concuerda con los resultados de la investigación de Sánchez-Martín (2020) sobre la influencias sociofamiliar en la elección de los estudios de Formación Profesional. Además, ambas titulaciones están altamente feminizadas y aunque las creencias de género no son un factor relevante en la elección.

Entre las conclusiones del segundo objetivo "Conocer los determinantes académicos en la elección de los títulos de grado de Educación Primaria y PCEO" destacan:

Los estudiantes de ambas titulaciones de grado acceden, principalmente desde Bachillerato, con una calificación de notable, aunque en el grupo de PCEO el rendimiento académico es levemente mayor. Resultados que coinciden con el estudio realizado por Martínez-Martínez et al., (2015) sobre la influencia de la modalidad de estudios, las calificaciones y el género en la elección del grado, el elevado número de estudiantes que han elegido estas titulaciones como primera opción sugieren que son titulaciones muy demandadas.

Los participantes que eligieron su titulación como segunda o tercera opción se debió a limitaciones de plaza, dudar, no poder acceder a dicha titulación en su ciudad, cambiarse de carrera o por considerar que tiene pocas salidas profesionales. Resultados que coinciden con los de autores como Martínez-Martínez et al. (2016) y Martínez-Martínez et al. (2015) quienes señalan que Magisterio es uno de los grados más demandados por no tener limitaciones económicas y familiares, pero que discrepan al considerar la nota de corte como limitación para obtener plaza.

La mayoría de encuestados declara no haber realizado ninguna prueba de orientación, destacando la falta de orientación previa recibida para la elección de los estudios de grado, que los participantes en este estudio consideran insuficiente e insatisfactoria. Aunque este hecho no ha afectado al grado de satisfacción con la carrera elegida, pues más de la mitad de los participantes se sienten muy satisfechos con su elección y entre sus expectativas de futuro está finalizar con la carrera. De esta manera los procesos de orientación son esenciales para hacer frente al abandono y garantizar el futuro laboral de los estudiantes. Por lo que, conocer las motivaciones, expectativas y demandas sociales puede contribuir a mejorar este proceso y contribuir a la reducción de los índices el abandono (Sánchez-Martín \& Contreras Mateos, 2018).

Generalizando los resultados obtenidos, los participantes tienen éxito académico, satisfacción con la carrera y estabilidad en su decisión, tres factores que para autores como Lauretti (2004, citado en Cepero González, 2009) determinan la elección de carrera.

Respecto al tercer objetivo "Describir los motivos para la elección de los títulos de grado de Educación Primaria y PCEO", se concluye que: 
Los determinantes personales intrínsecos son los que más peso tuvieron en la elección de ambas titulaciones. Resultados que coinciden con los obtenidos por Sánchez-Martín et al. (2017) sobre la elección del itinerario en Formación Profesional, quienes señalan que los principales determinantes que influenciaron dicha elección fueron los personales; así como con los resultados del estudio realizado por García-Ripa et al. (2018), según el cual las personas que cursan estudios sociales le dan menos valor a los motivos extrínsecos, predominando este tipo de motivación entre las féminas.

Los motivos que influyen en la elección de la carrera para los encuestados de Primaria son: en primer lugar, los personales intrínsecos, en segundo lugar los profesionales extrínsecos, en tercer lugar los profesionales intrínsecos y, en último lugar, los instrumentales extrínsecos son los menos valorados. Mientras que para los encuestados de PCEO son: primero los personales intrínsecos, a continuación, los profesionales extrínsecos, posteriormente los instrumentales extrínsecos y finalmente los profesionales intrínsecos. Este orden de preferencia corresponde con los resultados de García-Ripa et al. (2018) según el cual las personas que cursan estudios sociales les dan menos valor a los motivos extrínsecos, aunque entre los participantes los motivos profesionales extrínsecos son más valorados que los profesionales intrínsecos.

Entre las principales implicaciones educativas de este estudio, cabe destacar que el "proceso de orientación requiere la vinculación y la coordinación entre los Centros Educativos de Educación Primaria y Secundaria, y Bachillerato, con los Centros Universitarios" (Domínguez, Álvarez y López, 2013; Llosa, Acín, Cragnolino y Lorenzatti, 2012; citado en Martínez Martínez et al., 2015, p. 19). Por lo que es esencial continuar realizando investigaciones en las distintas titulaciones del ámbito universitario sobre los determinantes que afectan la elección del grado con sus alumnos de primer curso; para que, por una parte, los Centro mejoren sus test de orientación con los datos obtenidos en los distintos Grados universitarios, ya que como afirman Sánchez-Martín y Contreras Mateos (2018) se deben tener en cuenta las expectativas, motivaciones y demandas sociales para que esta orientación sea satisfactoria y las universidades puedan orientar su formación para cumplir con las expectativas de los estudiantes (stakeholders), mejorando su grado de satisfacción sobre esta elección. Contribuyendo a adaptar su servicio a las "personas directamente implicadas en la institución y cumplir así lo más eficazmente posible los objetivos que la sociedad democrática atribuye a la universidad" (Masjuan, 2005, p. 99). Por lo tanto, sería conveniente desarrollar esta conducta vocacional, el autoconocimiento y las autoevaluaciones, puesto que para autores como Cepero González (2009) las preferencias vocacionales son la combinación de estos dos últimos aspectos con los intereses y aspiraciones.

A lo largo del desarrollo de este trabajo nos hemos encontrado con una serie de limitaciones que hemos podido subsanar a lo largo de proceso de investigación, sin embargo, cabe destacar aquellas que no hemos podido resolver:

Por una parte, no haber podido realizar un muestreo probabilístico para que los datos fuesen generalizables. Como se señaló en el procedimiento esta limitación se debió al estado de alarma generado por el Covid-19, y aunque se intentó ampliar la muestra a través de cuestionarios on-line no hubo respuesta por parte de los estudiantes. Por lo que para futuras investigaciones nos planteamos ampliar este estudio con otras titulaciones universitarias comparando los resultados según ramas de conocimiento realizando muestreos probabilísticos representativos de la población objeto de estudio.

Por otra parte, los ingresos medios de la unidad familiar discrepan de el resto de los datos económicos de la unidad familiar, lo cual nos lleva a plantearnos la posibilidad de que los encuestados no hayan sido del todo sinceros en este ítem. Es decir, si el mayor 
rango de la unidad familiar está compuesto por cuatro miembros en el que trabajan dos de ellos (el $88 \%$ de los progenitores), los cuales poseen un nivel de estudios alto.

\section{Referencias}

Abreu, JL. (2012). Hipótesis, Método \& Diseño de Investigación. International Journal of Good Conscience, 7(2), 187-197.

Cepero González, AB. (2009). Las preferencias profesionales y vocacionales del alumnado de Secundaria y Formación Profesional Específica. (Tesis doctoral). Universidad de Granada, Granada.

García Garduño, JME. (2010). Los determinantes de la elección vocacional de una semiprofesión. Un estudio de estudiantes de primer ingreso a la carrera de profesor de Educación Primaria. Revista Latinoamericana de Estudios Educativos, 40(1), 95-110.

García-Ripa, MI., Sánchez-García, MF. y Risquez, A. (2018). Perfiles motivacionales de elección de estudios en estudiantes universitarios de nuevo ingreso. Universitas Psychologica, 17(3), 1-12.

Martínez Martínez, A. (2013). La orientación como actividad educativa y vocacional en los itinerarios curriculares del alumnado de bachiller y formación profesional y su inclusión en el mercado laboral. (Tesis doctoral, Universidad de Granada).

Martínez Martínez, A., Castro Sánchez, M., Lucena Zurita, M. y Zurita Ortega, F. (2015). Elección de titulación universitaria y expectativas de resultados de los adolescentes de Granada. Revista Española de Orientación y Psicopedagogía, 26(3), 63-77.

Martínez Martínez, A., Castro Sánchez, M., Zurita Ortega, F. y Lucena Zurita, M. (2015). La elección de estudios superiores universitarios en función de la modalidad de estudios, la nota media y el género. Magister, (27), 18-25.

Martínez-Martínez, A., Zurita-Ortega, F., Castro-Sánchez, M., Chacón-Cuberos, R., Hinojo-Lucena, MA. y Espejo-Garcés, T. (2016). La elección de estudio superiores universitarios en estudiantes de último curso de bachillerato y ciclos formativos. Revista Electrónica Educare, 20(1), 1-18.

Masjuan, JM. (2005). Progresos en los aprendizajes, características de los estudios y motivaciones de los estudiantes. Papers, (76), 97-133.

Rivas, F. (2003). Conducta y asesoramiento vocacional en la adolescencia. Papeles del Psicólogo, 23(84).

Romero Rodríguez, S. (2004). Aprender a construir proyectos profesionales y vitales. REOP, 15(2), 337-354.

Sánchez-Martín, M. (2020). Influencias sociofamiliares en la elección de los estudios de formación profesional. Revista Latinoamericana de Estudios de Familia, 12(1), 44-62. 
Sánchez-Martín, M., \& Contreras Mateos, Á. (2018). Construction and Validation of an Instrument: Factors That Influence Students' Decision to Choose a Superior Training Cycle. The International Journal of Diversity in Education, 18, 1-9.

Sánchez-Martín, M., González, C. y Martínez, P. (inédito). Validación del Cuestionario sobre Determinantes para la Elección del Grado Universitario (CDEGU).

Sánchez Martín, M., Izquierdo Rus, T. y Contreras Mateo, Á. (2017). Determinantes para la elección del itinerario formativo en el alumnado de formación profesional. Estudio presentado en XVIII Congreso Internacional de Investigación Educativa. Interdisciplinaridad y Transferencia, Salamanca.

Vega-Malagón, G., Ávila-Morales, J., Vega-Malagón, AJ., Camacho-Calderón, N., Becerril-Santos, A. y Leo-Amador GE. (2014) Paradigmas en la investigación. Enfoque cuantitativo y cualitativo. European Scientific Journal, 10(15), 523-528. 